\title{
O PAPEL DO ANESTESIOLOGISTA NO ATENDIMENTO DO TRAUMA
}

\author{
ANESTHESIOLOGIST'S ROLE IN TRAUMA MANAGEMENT
}

Alexandre Slullitel ${ }^{1}$; Angela Maria Sousa ${ }^{2} \&$ Adilson de Oliveira Fraga $^{3}$

\begin{abstract}
${ }^{1}$ Médico Assistente do Serviço de Anestesia do Hospital das Clínicas da Faculdade de Medicina de Ribeirão Preto - USP e Serviço de Anestesiologia do Instituto do Coração (InCor); ${ }^{2}$ Mestre em Farmacologia pelo Departamento de Farmacologia da FMRP-SUP e Médica Assistente do Serviço de Anestesia do Hospital das Clínicas da Faculdade de Medicina de Ribeirão Preto - USP; ${ }^{3}$ Docente da Faculdade de Medicina de Pouso Alegre, Médico Assistente do Serviço de Anestesiologia do Instituto do Coração (InCor), e da Divisão de Anestesiologia do Hospital das Clínicas da Faculdade de Medicina - USP.

CorrespondêncIA : Dr. Alexandre Slullitel. Rua: Adalberto Pajoaba, 957- Bloco C - apto. 24 - CEP: 14055-220 - Ribeirão Preto - S.P. E-mail : alexslullitel@uol.com.br
\end{abstract}

SLULLITEL A; SOUSA AM \& FRAGA AO. O papel do anestesiologista no atendimento do trauma. Medicina, Ribeirão Preto, 32: 251-261, jul./set. 1999.

RESUMO: O papel do anestesiologista, dentro da equipe de atendimento multiprofissional no trauma, tem aumentado progressivamente nos últimos anos. Esta revisão aborda o papel do especialista tanto em relação ao atendimento pré quanto intra-hospitalar até a fase de reabilitação do trauma. Métodos de controle da via aérea, reposição volêmica, monitorização e uso de drogas anestésicas no traumatizado são discutidos. A analgesia pós-operatória é apresentada como método de redução da morbimortalidade pós-operatória com diminuição dos custos de internação hospitalar .

UNITERMOS: Coração. Ressuscitação. Traumatologia. Hidratação. Anestesia.

\section{INTRODUÇÃO}

$\mathrm{O}$ atendimento ao politraumatizado deve ser multidisciplinar devido à repercussão de lesões sobre diversos sistemas orgânicos. No entanto, durante uma fase inicial, dos primeiros minutos às horas que sucedem ao trauma, preconiza-se a adoção de rotinas (algoritmos) de avaliação e tratamento, visando reduzir a morbidade e mortalidade. Tais algoritmos podem ser aplicados por qualquer profissional médico adequadamente treinado, engajado no atendimento de urgências. Progressivamente, o anestesiologista tem ocupado lugar de destaque no atendimento ao paciente politraumatizado.

No Brasil, tradicionalmente, a prática da anestesiologia esteve restrita à atuação em quirófanos, atendendo apenas a pacientes cirúrgicos no período intraoperatório. Na Europa, e particularmente na França, o anestesiologista, mais recentemente, é o responsável por todos os níveis de atendimento ao politraumatizado. Os Departamentos de Anestesia e Ressuscitação, Anestesia e Terapia Intensiva e Anestesia e Reanimação coordenam os serviços médicos de emergência pré e intra-hospitalares. Na França, os anestesiologistas têm atuação fundamental no SAMU (Service d'Aide Médicale Urgente - Serviço de Atendimento Médico de Urgência) que inspirou o nosso similar nacional. Nos Estados Unidos, criaram-se os Especialistas em Anestesia e Terapia Intensiva nos Traumatismos (EATIT) que, em alguns centros, são os responsáveis pela recepção do traumatizado transportado por helicóptero ou pelo pessoal de transporte da ambulância, a exemplo do que ocorre no Centro de Choque Traumático R. Adams Cowley, do Sistema de Serviços Médicos de Emergência do Instituto Maryland. A familiaridade do anestesiologista com pro- 
cedimentos que envolvem obtenção de vias aéreas complicadas e domínio de técnicas de ventilação mecânica, obtenção de acessos vasculares para instalação de infusões venosas, instalação de monitorização invasiva e não invasiva e transfusão sangüínea, entre outros, qualificam-no como elemento indispensável dentro de serviços de atendimento ao traumatizado. Desta forma, podemos destacar a atuação dos anestesiologistas em algumas áreas de interesse no atendimento ao traumatizado: fase pré-hospitalar, fase intra-hospitalar (indução e pré-indução, manutenção anestésica intra-operatória, hemoterapia e reposição volêmica, terapia anestésica peroperatória), terapia intensiva pós-operatória.

\section{ATENDIMENTO PRÉ-HOSPITALAR}

A principal controvérsia em relação ao atendimento pré-hospitalar do trauma diz respeito ao tipo de provedor de assistência pré-hospitalar e ao tipo de intervenção executada. Nos E.U.A. e Reino Unido, os serviços médicos de urgência empregam paramédicos. $\mathrm{Na}$ Europa, particularmente na França, Alemanha e Bélgica, as ambulâncias são assistidas por médicos, freqüentemente anestesistas, nas unidades móveis de terapia intensiva. No Reino Unido, os técnicos de ambulância são treinados para a execução do controle de vias aéreas, imobilização cervical e desfibrilação. Os paramédicos possuem habilidades de entubação traqueal, canulação venosa, reposição volêmica e utilização de analgesia por via endovenosa. Habilidades suplementares, prestadas por médicos, incluem ainda o uso de bloqueadores neuromusculares (curares) e um amplo espectro de analgésicos e de substitutos para reposição volêmica, drenagem torácica, cricotireoidotomia e a capacidade de triagem ao hospital mais apro$\operatorname{priado}^{(1)}$.

\section{CONTROLE PRÉ-HOSPITALAR AVAN- ÇADO DAS VIAS AÉREAS}

A importância do controle precoce da via aérea reside na possibilidade de oxigenar e ventilar adequadamente o paciente, para garantir boa oferta de oxigênio aos tecidos. A técnica ideal seria aquela que, de forma bem simples, permitisse ventilação eficiente, com mínimo risco de insuflação gástrica, sem efeitos adversos cardiovasculares, em pacientes hipovolêmicos ou com trauma craniencefálico (TCE), e que garantisse a estabilidade da coluna cervical. A entubação traqueal ainda é considerada a técnica padrão ("gold standard", do inglês), embora, muitas vezes, seja de difícil execução em sítio extra-hospitalar. Assim, outras técnicas alternativas têm sido utilizadas.

A ventilação sob bolsa-válvula-máscara, empregando-se as cânulas orofaríngeas (Guedel) ou nasofaríngeas pode ser melhor realizada com a ajuda de um segundo auxiliar e possui a possibilidade de insuflação gástrica e aspiração. O combitubo é um tubo de dupla via, desenhado para ventilar os pulmões quer o tubo penetre na traquéia ou no esôfago. É introduzido, às cegas, pela boca e posiciona-se no esôfago (90\% dos casos) ou na traquéia. Uma das luzes é uma sonda de entubação tradicional, a outra, um tubo obturador esofágico, fechado na extremidade distal e com múltiplas perfurações situadas acima do balonete distal. O balonete distal, uma vez insuflado, fixa-se ou sobre o esôfago ou sobre a traquéia. Um balonete proximal maior de $100 \mathrm{~mL}$ oclui as cavidades nasais e oral, quando insuflado. Está contra-indicado nos traumas orofaríngeo ou esofágico graves ${ }^{(2)}$. A máscara laríngea $(M L)$ permite ventilação rápida e eficiente com um único operador, com menos fadiga das mãos e menor risco de insuflação gástrica se comparada aos dispositivos de bolsa-válvula-máscara. A ML não protege completamente a via aérea do risco de aspiração. A freqüência de mau posicionamento da MLé bastante variável, porém representa uma técnica bem mais simples para pessoas menos habilitadas a realizarem entubação orotraqueal. A ML pode ser empregada, principalmente, em casos de encarceramento, quando o acesso ao paciente é limitado. Pode ser introduzida sem necessidade de flexão da cabeça e pescoço, mesmo estando-se posicionado de frente ao paciente. A introdução da ML provoca menos respostas cardiovasculares e menos elevações das pressões intracraniana (PIC) e intra-ocular (PIO). A ML pode oferecer algumas vantagens nos TCE e traumatismos penetrantes do globo ocular, nos quais a elevação da PIC e PIO, em resposta à laringoscopia e entubação traqueal, bem como a tosse e o "bucking", contribuem para a lesão cerebral secundária ou perda definitiva da visão. Um novo modelo de ML, a aramada (Fastrach $\left.{ }^{\circledR}\right)$, possui um formato especialmente desenvolvido para permitir a passagem de um tubo endotraqueal ou de um fibroscópio em seu interior, auxiliando nos casos de entubação difícil ${ }^{(3)}$. Os estiletes luminosos (Trachlight ${ }^{\circledR}$ ) por transiluminação dos tecidos moles, permitem ao operador referenciar-se pelo orifício da glote e ultrapassá-lo sem laringoscopia e sem mobilização da coluna cervical, introduzindo-se um tubo previamente montado sobre um guia ${ }^{(4,5)}$. 
Se o paciente possui uma obstrução da via aérea, e a entubação orotraqueal e a introdução da ML não são possíveis, a cricotireodotomia é um procedimento indicado. Alternativamente, surgem técnicas passíveis de utilização que estão relacionadas com a experiência pessoal com seu emprego. A entubação retrógrada pode empregar-se na ocorrência da via aérea difícil, prevista ou imprevista. As indicações para a entubação retrógrada são: 1) fracasso na tentativa de entubação orotraqueal (E.O.T.) com laringoscópio ou fibra óptica; 2) estabelecimento emergencial de via aérea, quando a visualização das cordas vocais é impedida por sangue, secreções ou alterações anatômicas; 3) quando houver necessidade, em casos de coluna cervical instável, na fratura de mandíbula, ou na presença de anomalia anatômica. As principais complicações da entubação retrógrada são: laringoespasmo, perfuração do esôfago, lesão de cordas vocais, edema de laringe, hemoptise, hematoma na submucosa da traquéia com obstrução distal, infecção pré-traqueal, traqueíte e fístula traqueal ${ }^{(6)}$.

Finalmente, embora a importância dos fibroscópios flexíveis seja limitada durante as manobras de ressuscitação, existem, recentemente, aparelhos portáteis, operados com pilhas (Rapiscope $\left.{ }^{\circledR}\right)$, que auxiliam a introdução do tubo endotraqueal ${ }^{(2)}$. Também podem ser empregados para confirmar o posicionamento adequado de um cateter passado através da membrana cricotireóidea, durante a obtenção de uma via aérea cirúrgica ${ }^{(7)}$.

Sabe-se, atualmente, que a regurgitação e a aspiração são consideradas as principais complicações durante as manobras de ressuscitação se as vias aéreas estão desprotegidas. A manobra de pressão sobre a cartilagem cricóide contra o esôfago subjacente, descrita por Sellick, em 1952, representa medida protetora até o controle efetivo das vias aéreas. Estudos indicam que uma pressão de 30 Newtons seja adequada e que a presença de uma sonda naso ou orogástrica pode aumentar a eficácia da pressão sobre a cricóide (manobra de Sellick). Tal manobra, no entanto, não é desprovida de problemas potenciais. Durante tentativas de ventilação com máscara facial, após a aplicação da manobra, ocorre redução do volume corrente expirado e elevação nas pressões de pico inspiratórias. Pode ocorrer obstrução completa da via aérea em até $10 \%$ dos casos. Pode ocorrer oclusão completa da via aérea se a pressão for aplicada em sentido caudal (que ocorre com paciente cuja cabeça encontra-se fletida por um travesseiro) devido ao efeito rotacional sobre as cartilagens aritenóides. A pressão pode ser realizada com uma das mãos ou com a segunda mão apoiando posteriormente a coluna cervical. Há indícios de que a técnica com uma única mão possa desalinhar a posição de neutralidade da coluna cervical, justificando o uso de um apoio posterior à coluna cervical. Se a entubação traqueal for difícil ou impossível, o uso de outros métodos alternativos, como a ML, pode ficar prejudicado na presença de pressão sobre a cricóide ${ }^{(2)}$.

A capnografia é considerada a técnica padrão para a confirmação do posicionamento adequado do tubo endotraqueal ou da permeabilidade da via aérea estabelecida por outros métodos. Os níveis de $\mathrm{CO}_{2}$ expirado podem estar reduzidos pela redução da oferta de gás carbônico aos pulmões, como aquela que ocorre durante a parada circulatória. Em virtude disso, a capnometria tem sido preconizada como método para avaliação da eficiência da ressuscitação cardio$\operatorname{pulmonar}^{(2,8)}$.

\section{REPOSIÇÃO VOLÊMICA PRÉ-HOSPI- TALAR}

Há controvérsias no que diz respeito à administração pré-hospitalar de fluidos. Estudos em animais e seres humanos sugerem que a pressão arterial sistólica possa ser mantida entre $50-70 \mathrm{mmHg}$ na fase de atendimento pré-hospitalar, sem prejuízo quanto ao prognóstico dos pacientes. Numa análise recente, em que pacientes foram subagrupados conforme otipo principal da lesão (trauma vascular, lesão de víscera sólida, lesão não cardíaca e lesão cardíaca), concluiu-se que apenas os traumas penetrantes do coração se beneficiaram com a reposição volêmica pré-hospitalar agressiva, implicando em redução da mortalidade intra-hospitalar. No entanto, este estudo causou divergências entre os traumatólogos. Parece um ponto de consenso que a obtenção precoce de acessos vasculares é benéfica, uma vez que não retarde a chegada ao hospital. No TCE e nos tempos pré-hospitalares prolongados, a administração de fluidos parece bastante lógica. No TCE, a hipotensão pode reduzir significativamente a pressão de perfusão cerebral na vigência de pressões intracranianas elevadas. No TCE grave, é adequado manter uma pressão arterial média (PAM) de $90 \mathrm{mmHg}$, caso assuma-se uma PIC de $20 \mathrm{mmHg}$, mantendo-se uma PPC > $70 \mathrm{mmHg}$ $(\mathrm{PPC}=\mathrm{PAM}$ - PIC). Assim, uma reposição volêmica inicial, suficiente para a manutenção das funções vi- 
tais, deve ser realizada até que o choque hemorrágico importante seja controlado de forma definitiva através de cirurgia. O objetivo inicial da reposição pode ser unicamente a obtenção de pulso periférico palpável. Após o controle cirúrgico da hemorragia, o objetivo da reposição volêmica passa a ser a otimização da oferta de oxigênio aos tecidos. Os demais aspectos da reposição volêmica serão abordados mais adiante ${ }^{(1)}$.

\section{ANALGESIA, SEDAÇÃO E BLOQUEIO NEUROMUSCULAR PRÉ-HOSPITALARES}

O tipo de sedação para a entubação pré-hospitalar de urgência não está definido na prática. Os protocolos do SAVT (Suporte Avançado de Vida no Trauma) desencorajam o uso de qualquer medicação sedativa que possa dificultar futura avaliação do paciente, quando de sua chegada ao hospital. No entanto, a disponibilidade de utilização de drogas de curta ação pode beneficiar alguns pacientes, minimizando efeitos deletérios, relacionados à entubação traqueal (taquicardia, hipertensão arterial, elevação da PIO e da PIC $)^{(9)}$. Também a promoção da analgesia pode ser feita com utilização de pequenas doses de analgésicos potentes ou através do uso de bloqueios regionais com anestésicos locais. Infelizmente, nem todas as drogas hipnoanalgésicas são familiares à classe médica em geral. Talvez, por isso, a prática de sedação e curarização para entubação seja mais comum nos países europeus onde o anestesiologista participa do atendimento pré-hospitalar. Assim, recentemente, foi sugerida a seguinte conduta, caso haja necessidade de entubação pré-hospitalar em um paciente consciente: monitorização (ECG, pressão arterial não invasiva, oximetria de pulso), pré-oxigenação (sistema de máscara acoplada a bolsa reservatório com alto fluxo de oxigênio durante três (03) minutos), sedação com etomidato $0.3 \mathrm{mg} / \mathrm{kg}$ E.V., curarização com succinilcolina $1 \mathrm{mg} / \mathrm{kg}$ E.V., manobra de Sellick logo após a perda da consciência (avaliada pela perda do reflexo corneopalpebral). A entubação deve ser realizada tão $\operatorname{logo}$ cessem as fasciculações ( 60 segundos) induzidas pela succinilcolina e a manobra de Sellick mantida até que o balonete do tubo endotraqueal seja insuflado (após confirmação do bom posicionamento da sonda). Utilizam-se opióides em pequenas quantidades para reduzir as respostas hemodinâmicas à entubação. $\mathrm{O}$ fentanil, em baixas doses de 1 a $2 \mu \mathrm{g} / \mathrm{kg}$ E.V., pode ser empregado com esse objetivo. A utilização desta técnica admite variações quanto ao tipo de agentes anes- tésicos empregados e se constitui no que os anestesiologistas convencionaram chamar de técnica de indução seqüencial rápida. A dosagem de fentanil, descrita acima, pode, também, ser empregada para a analgesia venosa em pacientes conscientes com queixa importante de dor. A realização de bloqueios regionais pode ser de difícil execução em campo ou por médicos não familiarizados com as diversas técnicas. A manutenção da sedação, durante o transporte, pode ser feita com midazolam infusão em contínua, a uma velocidade de $0.1 \mathrm{mg} / \mathrm{kg} / \mathrm{h}$. Essas medidas só devem ser adotadas quando uma avaliação inicial adequada do paciente for possível, de forma que o médico emergencialista possa, chegando ao hospital, informar a respeito das condições prévias do paciente (por exemplo, a classificação na escala de Glasgow). Na falha da entubação traqueal, após trinta (30) segundos de tentativa, deve-se prosseguir com a ventilação sob máscara, porém mantendo continuamente a pressão sobre a cricóide, a fim de prevenir a aspiração ${ }^{(10)}$.

\section{ANESTESIA NOS POLITRAUMATIZADOS}

Diferentemente dos pacientes programados para procedimentos cirúrgicos eletivos, o anestesiologista poucas informações tem a respeito da vítima do traumatismo e, em geral, desconhece as patologias que acometem, concomitantemente, os traumatizados. As principais informações dizem respeito aos mecanismos do trauma e às circunstâncias que se sucederam até a chegada do paciente ao centro cirúrgico. Duas considerações, porém, merecem destaque. A primeira delas é que o paciente politraumatizado é sempre considerado um paciente de "estômago cheio"; isto significa, que, independentemente de haver ingerido alimentos ou não na ocasião do trauma, existe grande risco de regurgitação ou aspiração do conteúdo gástrico. Isso é devido ao retardo do esvaziamento gástrico que pode ocorrer após o trauma. Assim, qualquer deterioração do nível da consciência coloca o paciente em risco de aspiração e, principalmente, durante a indução da anestesia geral ou realização de sedação profunda. Lembrar que, mesmo no caso de regurgitação de conteúdo gástrico, a coluna cervical deve sempre permanecer alinhada.

$\mathrm{O}$ outro aspecto a ser lembrado diz respeito à intoxicação por álcool e drogas. A intoxicação aguda ou crônica por álcool produz níveis elevados de nicotinamida-adenina-nucleotídeo reduzida (NADH), podendo causar hipoglicemia, cetoacidose alcoólica e aci- 
dose lática. As principais alterações hemodinâmicas são a vasodilatação periférica, depressão miocárdica e poliúria, que podem provocar redução da pressão arterial desproporcional à perda sangüínea real. A vasodilatação predispõe à hipotermia e ocorre redução da síntese de fatores de coagulação. Em relação às drogas, em nosso meio, a intoxicação mais freqüente é com a cocaína (principalmente o crack). A cocaína promove aumento das catecolaminas circulantes, provocando hipertensão, taquicardia, arritmias ventriculares, convulsões, midríase, hiperglicemia e hipertermia. Em centros, onde dosagens séricas de álcool e drogas são feitas de rotina, encontra-se uma positividade superior a $40 \%$ das amostras. O impacto destes fatores sobre a morbimortalidade ainda não foi adequadamente estudado ${ }^{(11)}$.

\section{MONITORIZAÇÃO INTRA-OPERATÓRIA}

A monitorização básica de qualquer paciente politraumatizado submetido à cirurgia de urgência inclui eletrocardioscopia (ECG), pressão arterial, temperatura corporal, débito urinário, concentração de $\mathrm{O}_{2}$ inspirado, $\mathrm{CO}_{2}$ expirado $\left(\mathrm{ETCO}_{2}\right)$ e oximetria de pulso $^{(12)}$

O ECG contínuo, em D II, permite o diagnóstico de arritmias e isquemia da parede inferior. Alternativamente, pode-se usar a derivação bipolar $\mathrm{CB}_{5}$ (eletrodo - posicionado sobre a escápula direita, posteriormente, e o eletrodo + sobre a posição de $\mathrm{V}_{5}$ ) capaz se detectar arritmias ou isquemia da parede anterior.

A medida da pressão arterial do paciente hipovolêmico através do método auscultopalpatório pode ser difícil e imprecisa. A medida da pressão arterial pelo método oscilométrico deve ser rotina, quando não for indicada a medida da pressão arterial de forma invasiva. A medida invasiva da pressão arterial é obtida por cateterização direta da artéria radial (mais freqüente). A medida da pressão arterial invasiva pode fornecer dados importantes em momentos críticos, como na indução da anestesia, no clampeamento e desclampeamento de grandes vasos ou nas hemorragias rápidas. O registro das ondas de pressão arterial permite avaliar o volume intravascular e, se ocorrer o pulso paradoxal, diagnosticar o tamponamento cardíaco. As mudanças cíclicas do traçado da pressão arterial, durante a ventilação com pressão positiva, são sugestivas de hipovolemia. Além do mais, o cateter arterial permite coletar amostras sanguiíneas sucessivas para exames laboratoriais.
A pressão venosa central (PVC) deve ser monitorizada quando está prevista grande administração de fluidos. Os anestesistas preferem a obtenção de um acesso via jugular interna, preferencialmente utilizando a técnica de Seldinger para a passagem de uma via introdutora para Swan-Ganz de 8. 5 Fr de diâmetro ou um cateter de dupla via. A PVC deve ser medida, preferencialmente, com transdutor de pressão, pois o manômetro de água, em pacientes taquicárdicos, não possui freqüência de resposta adequada e não permite a verificação rápida do posicionamento do cateter. É importante recordar que a PVC pode estar elevada no tamponamento cardíaco, contusões miocárdicas, pneumotórax e hemotórax, ainda que o paciente se apresente hipovolêmico.

O posicionamento de um cateter de artéria pulmonar (Swan-Ganz), durante a ressuscitação intra-operatória do paciente grave, requer tempo, mas pode ser benéfica em pacientes cuja história prévia indica disfunção ventricular, insuficiência coronariana importante ou valvulopatia. $\mathrm{O}$ cateter pode auxiliar no tratamento com pressão positiva expiratória final (PPEF) em pacientes que desenvolveram a síndrome do desconforto respiratório do adulto (SDRA), na diferenciação entre edema pulmonar de alta ou baixa pressão e no cuidado transoperatório de pacientes com trauma raquimedular e trauma torácico fechado, principalmente com contusão miocárdica associada. $\mathrm{O}$ posicionamento adequado do cateter, na zona 3 de West, é fundamental para a interpretação dos dados, o que nem sempre é possível em pacientes com diferentes tipos de lesão. É técnica invasiva com complicações freqüentes, que deve ser indicada quando os benefícios superam, em muito, os riscos ${ }^{(12,13)}$.

A diurese é um bom parâmetro da perfusão renal, mas esse método pode ser enganoso em situações de choque prolongado, insuficiência renal já estabelecida (poliúrica ou oligúrica) e devida à administração de contrastes radiopacos. Ocorre um retardo no tempo de resposta do fluxo urinário em relação à velocidade de reposição hídrica, reduzindo a eficácia do método. A diurese pode identificar a ocorrência de mio ou hemoglobinúria ${ }^{(12)}$.

A ecocardiografia transesofágica (ETE) tem sido utilizada por alguns centros para avaliação da volemia no intra-operatório, em substituição ao cateter de Swan-Ganz. A determinação do volume das câmaras cardíacas e da mobilidade da parede ventricular pode ser avaliada continuamente. No entanto, é tecnologia bastante cara que requer médicos adequada- 
mente treinados e não permite utilização em larga escala ao contrário do cateter de Swan-Ganz ${ }^{(12,14)}$.

A capnografia permite obter informações sobre a hipoventilação aguda, mau posicionamento do tubo traqueal, padrões anormais de respiração e embolia pulmonar ou aérea. A capnometria correlaciona-se diretamente com o débito cardíaco e permite avaliar, adequadamente, de forma não invasiva, as manobras de ressuscitação, inclusive a efetividade da massagem cardíaca. É imprescindível para a conduta intra-operatória de pacientes com TCE e lesão ocular.

A monitorização da temperatura é fundamental no politraumatizado, pois a indução da anestesia per se reduz a temperatura corpórea em até $1^{\circ} \mathrm{C}$, na primeira hora. O melhor local para a medida da temperatura, em pacientes traumatizados, ainda é objeto de controvérsia, porém as medidas das temperaturas timpânica, esofágica ou sangüínea (através do cateter de artéria pulmonar) refletem, aproximadamente, a temperatura central ${ }^{(12)}$.

A monitorização da função neuromuscular, por meio de estimulador de nervo periférico, deve ser usada em todos os pacientes submetidos à cirurgia de urgência por trauma, principalmente naqueles com TCE, lesão vascular tamponada e lesão do globo ocular na tentativa de evitar tosse, bucking, esforço abdominal e vômitos.

A oximetria de pulso é um método rápido, prático e contínuo de avaliar adequadamente a $\mathrm{SaO}_{2}$ (saturação arterial de oxigênio) entre 65 e $100 \%$. Porém, a técnica pode se tornar imprecisa, nos pacientes traumatizados devido à vasoconstrição periférica, hipotermia, aos níveis elevados de carboxiemoglobina devido a queimaduras e administração exógena de corantes (azul de metileno).

As gasometrias arterial e venosa mista, os níveis de hematócrito e hemoglobina, eletrólitos (sódio, potássio e cálcio) e a glicemia devem ser realizados na admissão do paciente e repetidos a intervalos regulares, conforme a necessidade do caso ${ }^{(12)}$. A avaliação da coagulação é necessária, principalmente nos pacientes politransfundidos e hipotérmicos. Um coagulograma completo inicial com tempo de protrombina (TP), tempo de tromboplastina parcial (TTPa), contagem de plaquetas, fibrinogenemia e produtos de degradação de fibrina (PDF) deveria ser obtido para rastreamento retrospectivo de coagulopatias já instauradas na admissão hospitalar. Uma alternativa mais viável pode ser a utilização do tromboelastograma, embora ainda faltem estudos sobre a aplicação deste método em pacientes politraumatizados ${ }^{(15)}$.

\section{TÉCNICAS ANESTÉSICAS}

A seleção quanto ao tipo de técnica anestésica (anestesia geral ou regional) depende de alguns fatores como: tipo das lesões, cirurgia planejada, ocorrência de hipovolemia, ocorrência de distúrbios de coagulação, história de doenças preexistentes e estado mental do paciente.

A anestesia regional, representada pelos bloqueios regionais, bloqueios de plexo braquial, anestesia raquidiana ou peridural, é preferida na vigência de lesões das extremidades ou do períneo. A anestesia regional deve ser a técnica de eleição uma vez descartados a hipovolemia e os distúrbios de coagulação, em virtude do menor risco de aspiração de conteúdo gástrico $^{(16)}$. Além disso, as técnicas regionais permitem uma avaliação permanente do estado mental. A anestesia geral está indicada nos pacientes com traumas cranianos, maxilofaciais, torácicos ou abdominais.

\section{AGENTES ANESTÉSICOS}

O agente anestésico ideal não existe. Seria aquele agente de início rápido de ação, pouco efeito depressor do miocárdio e mínima vasodilatação periférica. Os principais agentes hipnóticos são os barbitúricos (tiopental), a quetamina, o etomidato, os benzodiazepínicos (midazolam) e o propofol. O tiopental ainda é a referência para a indução em caso de estômago cheio. O tiopental provoca vasodilatação associada à redução do débito, motivos que justificam sua utilização em pacientes com pressão arterial quase normal e frequiência cardíaca inferior a $100 \mathrm{bpm}$. O tiopental é o hipnótico com mais rápido efeito de ação. A quetamina é o anestésico que promove a chamada anestesia dissociativa, ou seja, impede a percepção da dor de maneira consciente. A quetamina promove estimulação simpática com liberação de catecolaminas endógenas. Este fato torna-a atrativa para utilização em pacientes hipovolêmicos e hipotensos. Infelizmente, possui efeito depressor do miocárdio e, isoladamente, promove elevações da pressão intracraniana em pacientes com TCE, em ventilação espontânea. Em pacientes em choque prolongado, com depressão dos níveis de catecolaminas endógenas, seu uso provoca, predominantemente, depressão miocárdica. O etomidato é desprovido de grandes alterações cardiovasculares em pacientes normovolêmicos e, além disso, reduz a PIC e PIO. O propofol é um agente hipnótico que promove uma redução importante da resistência 
periférica que o torna desinteressante como droga hipnótica em pacientes hipovolêmicos. Porém promove redução da PIO e da PIC. É conveniente lembrar que os hipnóticos, como o propofol, reduzindo a pressão arterial, podem, também, reduzir a pressão de perfusão cerebral, apesar da redução da PIC. O midazolam é o benzodiazepínico mais empregado e sua utilização no trauma está associada a sua capacidade de induzir amnésia. Apresenta repercussão hemodinâmica em pacientes hipovolêmicos e possui início de ação lento, tornando-se pouco vantajoso para a indução no paciente com estômago cheio. Os opióides (alfentanil, fentanil e sufentanil) são drogas que apesar da excelente analgesia, não produzem nem amnésia e nem perda de consciência. Os opióides reduzem o tônus simpático basal e, assim, determinam efeitos indiretos sobre a resistência periférica e pressão arterial. O alfentanil possui o mais rápido efeito de ação e apresenta, portanto, maior interesse na indução seqüencial rápida ${ }^{(12)}$.

A succinilcolina é um relaxante muscular despolarizante, com rápido início de ação (sessenta segundos (60)) e curto duração de efeito (três (03) a cinco (05) minutos). As principais contra-indicações a sua utilização são doenças neuromusculares congênitas, síndrome de desnervação extensa, de ocorrência em uma semana após a instituição de paraplegia e com duração de cerca de seis (06) meses, queimaduras graves (> $30 \%$ área corporal total), politraumatizados com lesão muscular importante, déficit de pseudocolinesterase plasmática, alergia à succinilcolina, antecedentes familiares de hipertermia maligna. A succinilcolina é agente desencadeante da hipertermia maligna e está relacionada à ocorrência freqüente de reações anafilactóides, associadas aos curares em 25 a $50 \%$ dos casos. Provoca hipercalemia transitória, que tem sido responsabilizada por casos de parada cardíaca em pacientes queimados e politraumatizados, e deve ser evitada sobretudo nos pacientes com rabdomiólise. Entre os principais efeitos indesejáveis estão o aumento da PIC e da PIO. Estes efeitos podem ser atenuados pela administração prévia de agentes hipnóticos (exceto a quetamina) ou do manitol. Os relaxantes musculares adespolarizantes (pancurônio, atracúrio, vecurônio, rocurônio, mivacúrio) apresentam a vantagem de não compartilharem dos efeitos colaterais da succinilcolina. O rocurônio é um curare de duração de efeito intermediário, com rápido início de ação. As condições de entubação são similares à succinilcolina, após uma dose de 0.6-0.9 mg/kg E.V., porém a duração do efeito é duradoura ( > 30 minutos ), tornando seu uso desaconselhado em vista da possibilidade de difícil entubação imprevista. O rapacurônio (ORG 9487) é um curare adespolarizante de ação rápida, cuja duração do efeito é inferior à do rocurônio e demonstra condições de entubação semelhantes à da succinilcolina ${ }^{(17)}$.

Os agentes halogenados (halotano, enflurano, isoflurano, sevoflurano e desflurano) promovem redução da resistência periférica e redução da contratilidade miocárdica. Promovem aumento da PIC associado ao aumento do fluxo sangüíneo cerebral, caso sua administração não seja precedida por manobras que produzam hipocapnia. Devem ser evitados no TCE grave. $\mathrm{O}$ óxido nitroso $\left(\mathrm{N}_{2} \mathrm{O}\right)$ não deve ser empregado caso ocorra hipoxemia arterial com redução da oferta de oxigênio aos tecidos. Além disso, pode aumentar o tamanho de pneumotórax, pneumoencéfalo e pneumoperitôneo. Empregado isoladamente, pode provocar aumento importante do fluxo sangüíneo cerebral e, conseqüientemente, aumentos da $\operatorname{PIC}^{(12,18,19,20)}$.

\section{VENTILAÇÃO}

Algumas situações merecem destaque no manejo da ventilação, em pacientes politraumatizados. Basicamente, os distúrbios mais comuns estão associados à redução da capacidade residual funcional (CRF) e dificuldade de oxigenação. Em pacientes hipovolêmicos, a ventilação mecânica com pressão positiva pode comprometer o débito cardíaco e o retorno venoso, provocando redução da pressão arterial após cada ciclo respiratório. A diferença entre a pressão sistólica máxima e pressão sistólica mínima, durante cada ciclo respiratório, é um bom indicador de hipovolemia. Quanto maior o volume corrente administrado, maior a redução do débito cardíaco. Caso necessário, para aumentar a oxigenação arterial, pode-se utilizar a pressão positiva expiratória final (PPEF) com acréscimos sucessivos de 2.5 a $5 \mathrm{cmH}_{2} \mathrm{O}$ até haver melhoria da oxigenação arterial. A ventilação com PPEF até $30 \mathrm{~cm} \mathrm{H}_{2} \mathrm{O}$ não aumenta a PIC dos pacientes com TCE fechado, desde que um proclive de $30^{\circ}$ seja mantido, podendo, porém, provocar hipotensão por diminuição do retorno venoso e queda da PPC. Em pacientes com tamponamento cardíaco, com tórax fechado, a respiração espontânea mantém maior débito cardíaco do que a ventilação com pressão positiva, a qual causa maiores aumentos da pressão intratorácica e reduções do retorno venoso e débito cardíaco ${ }^{(12)}$. Embora o controle da PIC seja importante no TCE, 
não há, até o presente momento, evidências clínicas de que a hiperventilação melhore o prognóstico dos pacientes com TCE. Recomenda-se, atualmente, evitar a hiperventilação profilática no TCE e reservá-la para o tratamento da hipertensão intracraniana não controlada por outros métodos. Hiperventilação inadequada ou excessiva no intra-operatório pode reduzir ainda mais o FSC em cérebros com baixos fluxos sangüíneos normocapnéicos ${ }^{(21)}$.

\section{HIPOTERMIA}

A mortalidade no traumatizado tem associação direta com a intensidade da hipotermia pré e intraoperatória. Durante a cirurgia eletiva, a temperatura corpórea dificilmente cai abaixo de $34^{\circ} \mathrm{C}$ devido à ativação da vasoconstrição cutânea compensatória. No politraumatizado, a temperatura pode atingir valores inferiores a tal. A administração de soluções cristalóides, ou produtos hemoderivados, não aquecidas agrava e acelera o desenvolvimento da hipotermia. A hipotermia pode provocar redução do débito cardíaco, arritmias cardíacas, atenuação da vasoconstrição pulmonar hipóxica, redução dos fluxos sangüíneos cerebral e renal, alteração da função plaquetária e distúrbios hidroeletrolíticos. As principais maneiras de prevenir a hipotermia consistem em diminuir as perdas por radiação (temperatura da sala $>22^{\circ} \mathrm{C}$, cobertura do paciente com campos plásticos adesivos), convecção (redução da velocidade do ar condicionado da sala, utilização de sistemas de aquecimento por convecção de ar quente), condução (aquecimento das soluções de irrigação e dos líquidos intravenosos) e evaporação (umidificação e aquecimento dos gases inspirados, proteção das vísceras expostas com campos). Tais medidas devem ser mantidas no período pós-operatório até o reaquecimento completo do paciente ou a correção dos distúrbios induzidos pela hipotermia ${ }^{(12)}$.

\section{REPOSIÇÃO VOLÊMICA}

A adequação do volume intravascular, com restabelecimento da capacidade de transporte de oxigênio, e a reposição de fatores de coagulação são prioritárias na anestesia do paciente politraumatizado. $\mathrm{O}$ reconhecimento do grau de hipovolemia é baseado em dados clínicos, como pressão arterial, freqüência cardíaca, estado de consciência e débito urinário, além de valores laboratoriais de hematócrito e hemoglobina. A monitorização hemodinâmica invasiva auxiliará no tratamento da hipovolemia.
O debate sobre a preferência do uso de soluções cristalóides ou colóides ainda é tema atual. As soluções cristalóides são fluidos facilmente disponíveis que permitem a restauração rápida da volemia, ainda que às custas de discreta anemia dilucional inicial. O volume de cristalóides a ser infundido depende da perda volêmica estimada. A pressão coloidosmótica das soluções cristalóides, sendo menor que a das soluções colóides, tende a conferir-lhes um menor tempo de permanência no intravascular e, portanto, maior tendência a constituir o edema intersticial ou "terceiro espaço". Essa perda para o terceiro espaço reduz o volume intravascular e promove edema intersticial pulmonar, que pode comprometer a oxigenação. Ainda nenhum estudo controlado mostrou a vantagem de um tipo qualquer de solução em relação à morbidade e mortalidade dos pacientes $^{(22)}$.

A solução de Ringer-lactato é a preconizada para a reposição inicial do politraumatizado, pois infusões de grandes quantidades de solução fisiológica podem promover acidose hiperclorêmica. O Ringer-lactato deve ser evitado nos TCE, pois possui osmolaridade inferior à plasmática $(280 \mathrm{mOsm} / \mathrm{L})$, podendo agravar o edema cerebral.

A solução salina hipertônica a $7.5 \%$ tem sido empregada com bons resultados, pois infusão de um pequeno volume desta solução pode "recrutar" boa quantidade de líquido intersticial, aumentando a pressão arterial. A solução salina hipertônica mostrou-se benéfica numa subpopulação de pacientes com TCE, atendidos com uma pontuação de, no máximo oito (08) pontos na escala de coma de Glasgow, permitindo maior número de altas hospitalares das de pacientes que receberam solução de Ringer-lactato. A infusão deve ser limitada a um total de $4 \mathrm{~mL} / \mathrm{kg}$ e está associada a maior incidência de hipernatremia. A administração de grandes quantidades desta solução, em pacientes queimados, aumentou em quatro (04) vezes a incidência de insuficiência renal aguda e, em duas (02) vezes, a mortalidade ${ }^{(1)}$.

As soluções de gelatinas são soluções coloidais de baixo custo, que representam alternativa viável às soluções cristalóides. Não são aprovadas para uso nos Estados Unidos, porém são amplamente empregadas nos países europeus. Podem ser infundidas até um volume de $40 \mathrm{~mL} / \mathrm{kg} /$ dia. Não causam interferência com a provas cruzadas do sangue e provocam distúrbios de coagulação associados, exclusivamente, à hemodiluição que determinam. As gelatinas são peptídeos de colágeno que se interligam por pontes uréicas 
e atingem peso molecular de 8000 a $35000 \mathrm{Da}$. A meia-vida das moléculas é de aproximadamente cinco (5) horas e, ao final de vinte e quatro (24) horas, apenas $5 \%$ das moléculas restam no intravascular. A maior parte das moléculas são excretadas pelos rins. Possuem eficácia inferior às soluções de amido e ao dextran 40. Aumentam o débito cardíaco por expansão da volemia e por hemodiluição e aumentam o índice cardíaco em $20 \%$, porém tais efeitos são efêmeros. São responsáveis por reações anafilactóides em freqüência um pouco superior aos amidos ${ }^{(23)}$.

O hidroxietilamido (HEA) é um polímero sintético, derivado da amilopectina. Diferentes graus de substituição de grupos hidroxietil pela glicose resultam em soluções de diferentes propriedades. O HEA possui peso molecular que varia entre 450000 e 200000 Da. Alguns derivados possuem uma meia-vida superior a $24 \mathrm{hs}$. Pode gerar coagulopatia por efeito no fator VIII e no fator de von Willebrand. O pentamido (200000 Da e grau de substituição de 50\% - HEA 200/0.5) possui uma meia-vida de 6 hs. Uma solução a $10 \%$ de HEA 200/0.5, utilizada em pacientes traumatizados ou sépticos, mostrou perfil hemodinâmico superior à solução de albumina humana a 20\%. Também podem induzir reações anafilactóides, embora com frequiência menor do que as gelatinas e o dextran. $\mathrm{O}$ volume de infusão recomendado é de $20 \mathrm{mg} / \mathrm{kg} / \mathrm{dia}$ das soluções de maior peso molecular. Possuem um custo elevado, porém inferior ao da albumina ${ }^{(24,25)}$.

Sistemas de infusão rápida de volume (Level One ${ }^{\circledR}$, Haemonetics-RIS $\left.\AA\right)$ foram projetados para permitir a administração de cristalóides ou colóides a velocidades entre 600 a $1200 \mathrm{~mL} /$ minuto, dependendo da viscosidade da solução infundida. Alguns sistemas permitem que as soluções infundidas mantenham-se aquecidas durante a infusão rápida. Também em casos de traumatismos torácicos e/ou na suspeita de lesão miocárdica e de grandes vasos, podem-se utilizar sistemas de recuperação de sangue intra-operatório, retransfundindo o sangue coletado no próprio indivíduo no período intra ou pós-operatório, sendo o objetivo a manutenção do hematócrito do paciente em cerca de $30 \%{ }^{(12)}$.

Os objetivos atuais da ressuscitação volêmica nos pacientes politraumatizados graves são aumentar o índice de oferta de oxigênio $\left(D_{2} \mathrm{I}\right)$ com reposição de fluidos e doses de dobutamina até $20 \mu \mathrm{g} / \mathrm{kg} / \mathrm{min}$ enquanto se monitorizam a saturação venosa mista de oxigênio $\left(\mathrm{SvO}_{2}\right)$, o déficit de base e/ou a concentração de lactato sérico ${ }^{(1)}$. No entanto, o uso da monitorização contínua do $\mathrm{pH}$ da mucosa gástrica $(\mathrm{pHi})$ - a tonometria capnométrica de gás recirculante - tem-se mostrado como bom índice da perfusão esplâncnica e tem-se correlacionado, adequadamente, com o prognóstico dos pacientes ${ }^{(26)}$.

\section{ANALGESIA PÓS-OPERATÓRIA}

A analgesia pós-operatória permite adequado controle da dor, reduzindo complicações pós-operatórias com mínimos efeitos colaterais. Reduzem-se as complicações pulmonares (atelectasias, pneumonias), as respostas endógenas ao estresse, a incidência de isquemia miocárdica, de trombose venosa profunda e embolia pulmonar ${ }^{(27)}$. Recentemente, observou-se que o controle adequado da dor permite não apenas um conforto muito maior do paciente, mas, também, a redução dos custos hospitalares relacionados com menor período de permanência hospitalar e em unidades de tratamento intensivo. A via de administração de analgésicos depende do tipo e local da lesão, bem como do nível de consciência do paciente. As principais vias de administração de analgésicos no pós-operatório são: oral, intramuscular, intravenosa (contínua, intermitente, controlada pelo paciente), epidural (contínua, intermitente, controlada pelo paciente), interpleural, intercostal e axilar (bloqueio do plexo do membro superior). Durante a reabilitação, analgesia adequada permite otimizar os cuidados fisioterápicos e o tratamento médico de complicações ou seqüelas com motivação do próprio indivíduo.

Os opióides são analgésicos potentes que, atuantes no sistema nervoso central e medula espinhal, são capazes de induzir analgesia pós-operatória intensa. Infelizmente, devido ao medo de ocorrência de alguns efeitos colaterais, como a depressão respiratória, são erroneamente subutilizados. Podem ser empregados por todas as vias referidas anteriormente, porém são mais comumente administrados por via intravenosa ou epidural. Sabe-se que a administração de opióides fora do ambiente da UTI ou da recuperação anestésica é muito segura e eficaz. Na técnica de analgesia controlada pelo paciente (ACP), o doente recebe pequenas doses de opióides através do acionamento de uma bomba de infusão eletrônica que infunde uma quantidade pré-determinada de analgésico, quando sentir dor. Um dispositivo interno da bomba impede a administração de doses consecutivas de analgésico em intervalo pré-estabelecido de cinco (5) a vinte (20) minutos até que a primeira tenha exercido seu efeito. $\mathrm{O}$ principal fator limitante ao seu uso, talvez, seja o custo das bombas ou dos equipos especiais, empregados 
nestes sistemas. As principais vantagens, no entanto, são o melhor controle da dor, menor sedação, melhor resultado dos testes de função pulmonar e a melhor adesão do paciente ao tratamento.Os principais opióides empregados são a morfina, a meperidina, o fentanil e o sufentanil ${ }^{(28,29,30)}$.

Os bloqueios intercostais, com anestésicos locais de longa duração, (bupivacaína) produzem analgesia eficaz após fraturas de costela, toracotomias ou incisões no flanco ou andar superior do abdômen. $\mathrm{O}$ principal incoveniente desta técnica está associado à necessidade de realização de bloqueios a curtos intervalos de tempo (seis a doze horas) e ao risco aumentado de intoxicação por anestésicos locais.

O bloqueio axilar, após o traumatismo da extremidade superior, proporciona alívio da dor e preservação da função motora. Um cateter para administração contínua ou intermitente de anestésicos locais pode ser posicionado por punção percutânea dentro da bainha do plexo braquial, prolongando o período de analgesia.

A analgesia epidural permite um controle da dor especialmente após ferimentos fechados do tórax ou após cirurgias torácicas ou abdominais altas, que limitam a função pulmonar e diafragmática. A associação de anestésicos locais e opióides, por via epidural, possibilita melhor controle da dor, com redução de efeitos colaterais. Estudos mostram redução da morbimortalidade em pacientes de risco com trauma torácico após utilização desta técnica. Assim, quanto maior o risco cirúrgico do paciente, maiores os benefícios da utilização da analgesia pós-operatória. As principais contraindicações relativas ao uso de analgesia epidural incluem hipotensão, sepse, infecção no local de punção e coagulopatia. Nas lesões vasculares ou dos membros inferiores, é conveniente discutir com a equipe cirúrgica a necessidade de monitorizar a função nervosa; neste caso, evitam-se os anestésicos locais por via epidural. O tempo de permanência do cateter epidural não se encontra bem estabelecido, mas, em situações especiais, estende-se por até sete (7) a catorze (14) dias , caso não ocorram sinais de infecção. Invariavelmente, o cateter se desloca do espaço epidural com a própria movimentação do paciente muito antes desse intervalo. A tunelização subcutânea do cateter epidural dificulta sua movimentação e protege contra infecções. O espaço epidural, de forma diferente do espaço intravascular, possui uma alta concentração de macrófagos que auxiliam no controle de infecções.

Os antiinflamatórios não esteroidais (AINES) não estão recomendados como analgésicos de escolha no trauma. Em nosso meio, talvez sejam empregados, erroneamente, de forma muito freqüente, na fase aguda do trauma. Estes medicamentos inibem a função plaquetária e podem determinar sangramento e, em pacientes hipovolêmicos, podem contribuir para a instalação de insuficiência renal. São drogas que apresentam rápida limitação da intensidade da analgesia, apesar do aumento das doses (efeito teto) e podem ser utilizadas posteriormente numa fase de recuperação ${ }^{(31)}$.

As seqüelas tardias, decorrentes da lesão de nervos somáticos, são particularmente freqüentes nos traumatizados. O reconhecimento precoce, assim como seu tratamento de forma agressiva, confere melhor prognóstico para a abordagem tardia de tais lesões. Entre as lesões iniciais, que evoluem para problemas crônicos, podemos citar a fratura múltipla de costelas, com lesão de nervos intercostais, a dor miofascial, as cefaléias pós-traumáticas por lesões "em chicote" da coluna cervical, as dores na articulação temporomandibular após trauma facial, dor por lesão da medula espinhal (principalmente nas lesões incompletas), a dor em "membro fantasma "após amputação traumática de extremidades, a dor por avulsão do plexo braquial (evoluem para as distrofias simpáticas reflexas). Todas estas causas tevem ter diagnóstico e tratamento precoces e estabelecem a importância da atuação do anestesiologista na fase de reabilitação do trauma ${ }^{(32)}$.

SLULLITEL A; SOUSA AM \& FRAGA AO. Anesthesiologist's role in trauma management. Medicina, Ribeirão Preto, 32: 251-261, july/sept. 1999.

ABSTRACT: The role for the anesthetist in multiprofessional trauma team has increased in the last few years. This paper reviews the role of the specialist concerning the pre-hospital and hospital management extended to the rehabilitation period. Methods of airway management, fluid therapy, monitoring and anesthetic drugs use are discussed. Postoperative analgesia as a way to reduce postoperative morbidity and mortality and reduce hospital stays costs is shown.

UNITERMS: Heart. Resuscitation. Traumatology. Fluid Therapy. Anesthesia. 


\section{REFERÊNCIAS BIBLIOGRÁFICAS}

1 - NOLAN JP \& PARR JA. Aspects of resuscitation in trauma. Br J Anaesth 79: 226-240, 1997.

2 - GABBOTT DA \& BASKETT PJF. Management of the airway and ventilation during resuscitation. Br J Anaesth 79: 159$171,1997$.

3 - BRIMACOMBE JR; BERRY AM \& BRAIN AIJ. Máscara laríngea. Clin Anest Am Norte 2: 389-415, 1995.

4 - HUNG OR \& MURPHY M. Bastões de luz, estiletes iluminados e técnicas de intubação às cegas. Clin Anest Am Norte 2: 455-467, 1995.

5 - SULLIVAN CA \& GOTTA AW. Problemas no reconhecimento e no controle da via aérea nos traumatismos. Clin Anest Am Norte 1: 13-28, 1996.

6 - SANCHEZ TF. Intubação Retrógrada. Clin Anest Am Norte 2: 417-454, 1996.

7 - MELKER RJ \& FLORETE Jr OG. Cricotirotomia: Revisão e discussão. Clin Anest Am Norte 3: 549-570, 1995.

8 - CROSBY ET. Controle da via aérea nos pacientes traumatizados. Clin Anest Am Norte 3: 637-658, 1995.

9 - KIL HK; BISHOP MJ ; BEDFORD RF. Respostas fisiológicas e fisiopatológicas à intubação. Clin Anest Am Norte 2: 341-355, 1995.

10 - ADNET F; HENNEQUIN B \& LAPANDRY C . Induction anesthésique en séquence rapide pour l'intubation trachéale préhospitalière. Ann Fr Anesth Réanim 17: 688-698, 1998.

11 - DOMSKY MF \& DOW AAC. Considerações anestésicas. Clin Ter Intens 4: 723-739, 1993

12 - CAPAN LM ; GOTTLIEB G \& ROSENBERG A . General principles of anesthesia for major acute trauma. In: CAPAN LM; MILLER SM \& TURNDORF H, eds. Trauma: Anesthesia and intensive care, J. B. Lip-pincott, Philadelphia, p. 259318, 1994.

13 - KARLIN A. Monitorização hemodinâmica dos pacientes traumatizados durante a anestesia. Clin Anest Am Norte 1: 85-99, 1996.

14 - HANOWELL LH; ANDERSON JT; KRAUT EJ \& BEAR JD. Transesophageal echocardiography in the perioperative assesment of intravascular volume. Sem Anesth Per Med Pain 17: 252-266, 1998.

15 - KAHN RC. Coagulation abnormalities and their management in the injured. In: CAPAN LM; MILLER SM \& TURNDORF $\mathrm{H}$ eds. Trauma: Anesthesia and intensive care, J. B. Lippincott, Philadelphia, p. 207-217, 1994.

16 - ROSENBERG AD \& BERNSTEIN RL. Anestesia regional e traumatismo. Clin Anest Am Norte 1: 101-123, 1996.

17 - WRIGHT PMC et al. A pharmacodynamic explanation for rapid onset/offset of rapacuronium bromide. Anesthesiology 90: 16-23, 1999.
18 - VIVIAND X; BOISSINOT P; DUBOULOZ F \& GRANTHIL C. Anesthésie-Reanimation du Traumatisé Gra-ve. In: Editions Techniques - Encyclopédie Médico-Chi-rurgicale. Paris. Anesthésie-réanimation, $36725 \mathrm{C}^{10}, 1992$.

19 - CARLI P \& ORLIAGUET G. Médicaments Anesthésiques et Choc Hémorragique. In: RIOU B ed. Journées d'enseignement post-universitaire d'anesthésie et de réanimation - Anesthésie en urgence. Arnette, Paris, p. 121-129, 1991.

20 - LAM AM \& MAYBERG TS. Anesthetic management of patients with traumatic head injury. In: LAM AM ed. Anesthetic management of acute head trauma. McGraw-Hill, New York, p. 181-221, 1995.

21 - BRIAN JE. Carbon dioxide and cerebral circulation. Anesthesiology 88: 1365-1386, 1998

22 - PROUGH DS. Cristalóides versus colóides no período peroperatório. Clin Anest Am Norte 2: 329-349, 1996.

23 - CONSEILLER C \& D’ENFERT J. Substituts colloïdaux du plasma. In: Editions Techniques - Encyclopédie Médico-Chirurgicale. Paris. Anesthésie-réanimation, $36735 A^{10}, 1983$.

24 - WARREN BB \& DURIEUX ME. Hydroxyethyl starch: Safe or not? Anesth Analg 84: 206-212, 1997.

25 - TREIB J \& BARON JF. Hydroxyéthylamidons: Effets sur I'hémostase. Ann Fr Anesth Réanim 17: 72-81, 1998.

26 -KNICHWITZ G ; VAN AKEN H \& BRUSSEL T. Gastrointestinal monitoring using measurement of intramucosal $\mathrm{PCO}_{2}$ Anesth Analg 87: 134-142, 1998.

27 - BALLANTYNE JC; CARR DB; deFERRANTI S ; SUAREZ T; LAU J; CHARMERS TL; ANGELILLO IF \& MOSTELLER F. The comparative effects of postoperative analgesic therapies on pulmonary outcome: Cumulative meta-analyses of randomized, controlled trials. Anesth Analg 86: 598-612, 1998.

28 - CHOQUET O; ROCHWERGER \& GUITOUKOULOU M. Analgésie périopératoire en chirurgie orthopédique et traumatologique. Cah Anesthesiol 46: 317-325, 1998.

29 - VASLEF SN \& VENDER JS. Cuidados pós-operatórios no paciente traumatizado. Clin Anest Am Norte 1: 239-256, 1996.

30 - BRUELLE P; VIEL E \& ELEDJAM JJ Bénéfices -risques et modalités de surveillance des différentes techniques et méthodes d'analgésie postoperatoire. Ann Fr Anesth Réanim 17: 502-526, 1998.

31 - SOUTER A; FREDMAN B \& WHITE PF. Controversies in the perioperative use of nonsteroidal antiinflammatory drugs. Anesth Analg 79: 1178-1190, 1994.

32 - RAUCK R. Trauma. In: RAJ PP ed. Pain medicine: A comprehensive review. Mosby, St Louis, p. 346-358, 1996.

Recebido para publicação em 11/08/99

Aprovado para publicação em 14/09/99 